\title{
Convoy: An Android App for Visually Challenged People
}

\author{
Pallavi Patil \\ Student of BE \\ Computer Dept. \\ K.J. Somaiya College \\ Of \\ Engineering \\ Mumbai, India
}

\author{
Revati Mulay \\ Student of BE \\ Computer Dept. \\ K.J. Somaiya \\ College Of \\ Engineering \\ Mumbai, India
}

\author{
Vrunda Shah \\ Student of BE \\ Computer Dept. \\ K.J. Somaiya \\ College Of \\ Engineering \\ Mumbai, India
}

\author{
Prasanna Shete \\ Assistant Professor \\ Computer Dept. \\ K.J. Somaiya College \\ Of \\ Engineering \\ Mumbai, India
}

\begin{abstract}
Traveling is integral task in our day today life. Visually challenged people face various challenges to travel alone. Our project, Convoy is Android application developed for navigation to assist visually challenged for traveling and security. The main scope is to provide interactive interface for visually challenged people and to help them while traveling in familiar and unfamiliar environments independently and safely using Global Positioning System (GPS) .The user will enter destination by vocal commands and app will give voice directions for navigation. When the user suspects danger or insecure situation, he can send SMS (Short Message Service) to the registered contact with its current location immediately. Thus, the use of the application will surely ease some of the difficulties faced by visually challenged user and can help them in achieving an independent livelihood.
\end{abstract}

\section{Keywords}

Convoy, Global Positioning System (GPS), SMS

\section{INTRODUCTION}

The ability to navigate from one place to another is an integral part of daily life for this vision plays a critical role but it would be difficult for visually impaired people. Although it would be easier to go to a familiar environment without vision but navigating to unfamiliar place without vision is very difficult. In spite of this, the visually challenged people travel to different places independently on a daily basis. But to facilitate safe and efficient navigation, visually challenged individuals must be guided properly.

Now-a-days, mobile phone is one of the most powerful entities in the world; ithelps for communication and to ease our day-to-day tasks. The existing application for visually challenged guidance like 'TalkBack'[1] provide the basic features for the accessibility of the mobile phone but the interaction is only one way as the application only speaks the actions. The navigation applications like Google Maps, Mapfactor[2] are designed to help common people for navigation. Thus we have designed 'Convoy', a complete navigation application for visually challenged people. This android application integrates accessibility with navigation and features for safety of the user. The main aim is to guide visually impaired people to travel from source to destination. The user gives input vocally and he is guided through audio commands. We have included a safety feature where user is able to send an alert message with his current location to registered contacts. The CONVOY android app will help visually challenged people to travel independently

\section{RELATED WORK}

K.Magatani[3] used digital map database in the system including road condition and route. To acquire exact position information map matching processing was performed. Similar digital map is used by author Koji Soeda[4] in there paper. Later advanced technologies like GPS were introduced which we have used in our app. In our project, we have integrated Google maps which are accurate and dynamic. SathiamoorthyManoharan[5] stated location visualization as how GPS location data is received in NMEA format. NMEA format needs to be translated for extracting information. Thus, we have developed a system that receives GPS data in the form of latitude and longitude, address of the location is extracted from latitude longitude information, thus current location is sent via SMS.

A. Al-Mazloum, E. Omer, M. F. A. Abdullah [6] propose system of GPS and SMS based child tracking using smart phone. It has client server architecture and parent is able to track the location of child by receiving SMS with GPS location of child. J. Snchez and F. Aguayo[7], uses 9-button virtual keyboard and also text to speech technology to get input from the user for their messenger system. Our project is in Android so various functionalities like sending SMS, text to speech ispresents. The developer has to integrate them in their application. BlindGuide [8] application does not require touch screen but 4 way navigation key where user can call by number or name. the voice enabled mobile application, SwarSuchak[9] was developed for information retrieval in many languages.

\section{APPLICATION DEVELOPMENT 3.1 Requirements}

The main component of the Convoy project is the software application, which will be limited to the Android operating system (specifically Android 4.3 (Jellybean) and above). The smart phone must be GPS enabled for proper navigation and network provider is required to send SMS. Internet is required to get current location and navigation.

\subsection{Application Architecture}

The CONVOY navigation android application is built considering visually challenged people. Along with assistance for navigation it also helps when the person is in danger, so that the location of the person could be sent. The system architecture requires internet and GPS enabled. For searching of the location on Google maps, app will require internet connection and data will be received from Google API server. 
The current location will be received from GPS locatorenabled in the cell phone. The SMS will be sent using the GSM network to the registered contact number via mobile network operator. The user will enter destination by speech, thus text to speech an speech to text module will be provide the necessary interaction

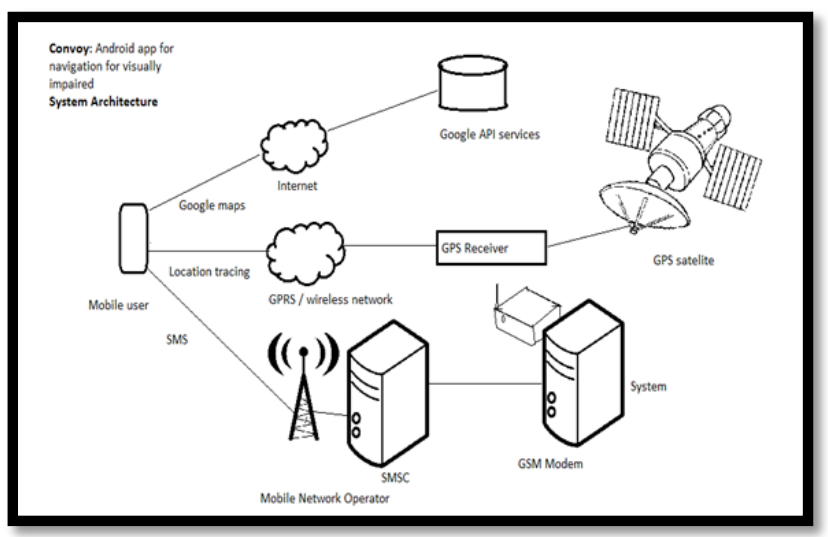

Fig 1: Architecture diagram for CONVOY

\section{IMPLEMENTATION}

The application assists the user in navigating to the destination from his current location. The user enters his destination and it is converted from speech-to-text by the system. The destination and current location are traced by the GPS in the smart phone of the user and using the services provided by the Google APIs, the directions from the source to the destination are acquired.

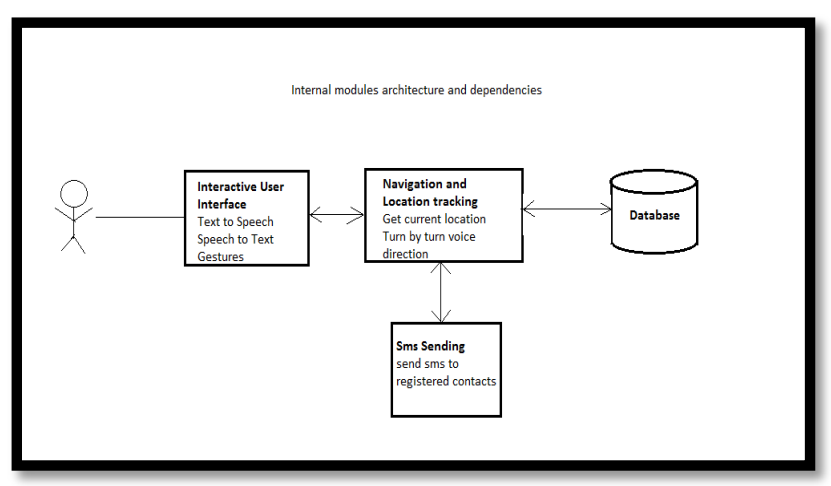

Fig 2: Internal modules of the project

\subsection{Interactive User Interface}

This is main module of the project. The text to speech and speech to text conversion is used to make application two-way interactive. As user press button, he could hear audio command "Enter destination", then user has to speak destination which will be stored for navigation. The navigation module will help user to navigate with help of vocal commandsthus visually challenged user can use our application effectively.

Android app development has various packages and functions which help to execute various functionalities in our application. The text to speech conversion is done using speak() function and creating object of TextToSpeech class[12].Speech to text module requires android.speech.RecognizerIntent package.

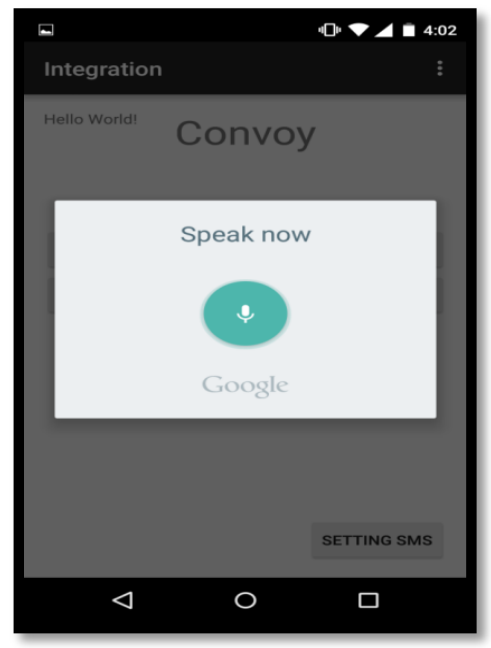

Fig 3: Recording of destination

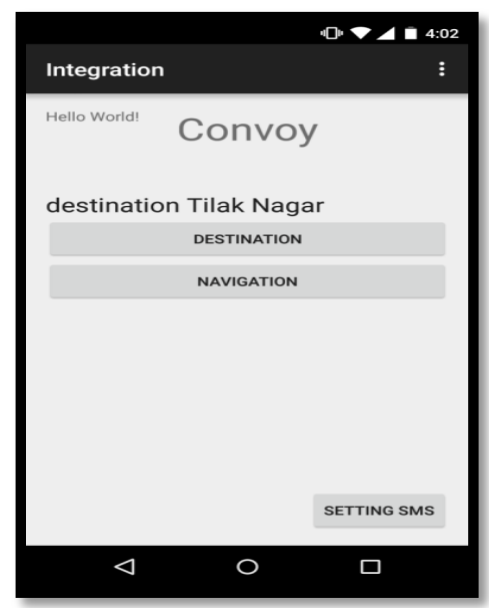

Fig 4: Destination recorded

\subsection{Navigation Module}

The navigation module gives turn by turn voice direction to the user. The user speaks source and press 'Navigation' button or taps with single finger twice, the navigation starts. This module requires internet connection and GPS enabling for proper direction through Google maps. The url is passed to get voice navigation appending the current location's and destination location's latitude and longitude data.

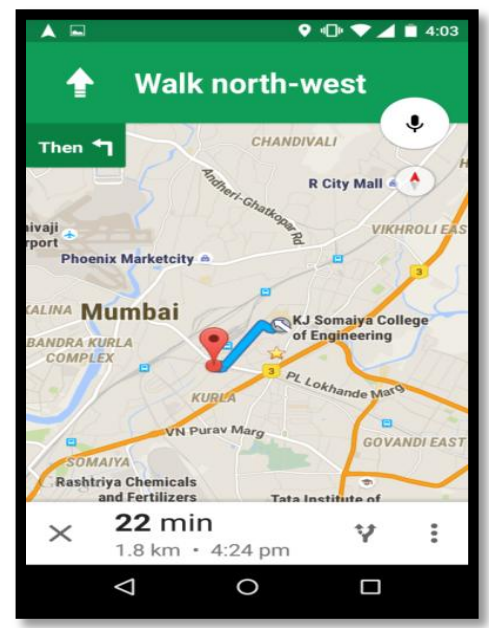

Fig 5: Navigation from source to destination 


\subsection{SMS sending module}

The user might sometime feel that something is suspicious then the user can send the location not only just with the latitude and longitude but also with the location address to the predefined contacts which are maintained in the database. This location would be sent to their predefined contacts by pressing on SMS button on interface, so that it would be much quicker rather than entering again into the system then searching the contact list and then sending it. The current location is retrieved by using class LocationManager and LocationListener. The address is extracted from latitude and longitude using Geocoder class. The contacts are retrived from the database and SMS is sent using SMSManger class from android.telephony.SMSManager package.

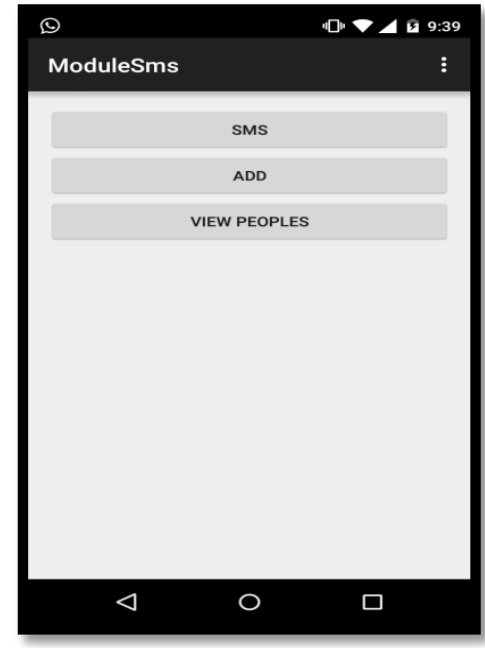

Fig 6: SMS button on interface

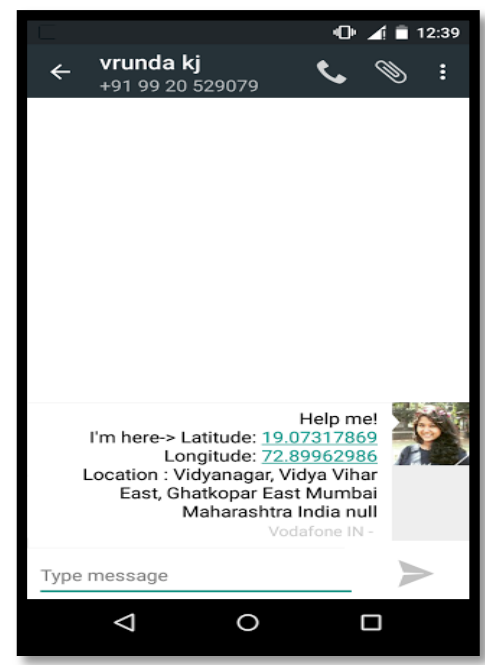

Fig 7: SMS sent with current location

\subsection{Database Module}

The database stores the name and contact details of the users well-wishers. The gesture will directly send SMS to these contacts so that they can rescue user in difficulty. SqLitedatabase[13] is created to store data. The user can add data using gesture and speech commands. The method used to create database is openOrCreateDatabase() and to execute query is exesQL(query).Thus, android.database.sqlite.SQLiteDatabase package is used.database.sqlite.SQLiteDatabase package is used

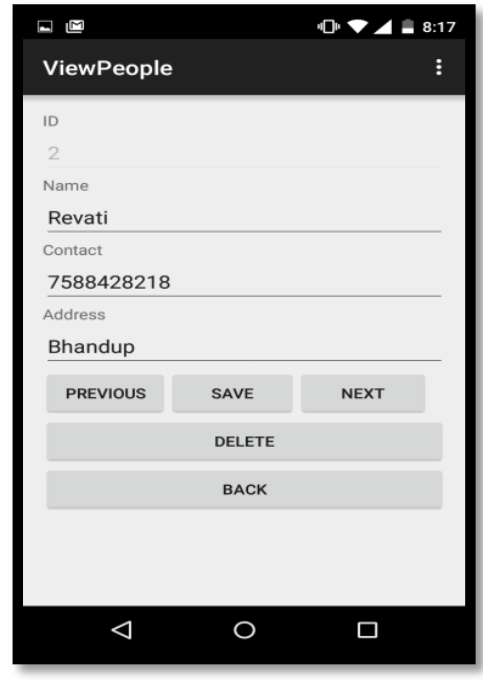

Fig 8: View of database content

\section{CONCLUSION}

We have presented an Android application for the assistance of the visually challenged user that will guide the user in navigating from his source to destination as well as it will help him in acknowledging his contacts about his location. The app was developed and Google Maps were integrated to search different location on map. SMS sending module is integrated to app, on triggering SMS button SMS to registered user is sent with user's current location. The application requires internet connectivity and GPS enabled smart-phone and thus can be easily accessed by the user. Thus the developed application is more accurate than the existing systems. The use of the application will surely ease some of the difficulties faced by visually challenged user and can help them in achieving an independent livelihood.

\section{FUTURE WORK}

The navigation is using Google map API, to launch this app on play store proper license must be obtained. The noise can be constraint so mechanism can be implemented to suppress or remove noise.

\section{ACKNOWLEDGEMENTS}

We would like to thank our mentor for his continuous guidance and support for the project and all professors of our institute. We also take this opportunity to thank our family and friends for their support and blessing.

\section{REFERENCES}

[1] https://play.google.com/store/apps/details?id=com.googl e.android.marvin.talkback\&hl=en

[2] https://www.mapfactor.com/en/

[3] H.Hashimoto, K.Magatani et al "The Development of the navigation system for the visually impaired persons", Proceedings of 23rd Annual International conference of IEEE Engineering in Medicine and Biology Society, 2528 Oct. 2001.

[4] Koji Soeda, Shingo Aoki, Kenji Yanashima, Kazushige Magatani, "Development of the visually impaired person guidance system using GPS", $26^{\text {th }}$ Annual International Conference of IEEE EMBS, San Francisco, CA, USA, 15 Sept. 2004. 
[5] SathiamoorthyManoharan,"On GPS Tracking of Mobile Devices", 20-25 April 2009 Fifth International Conference on Networking and Services.

[6] A. Al-Mazloum, E. Omer, M. F. A. Abdullah,"GPS and SMS-Based Child Tracking System Using Smart Phone", World Academy of Science, Engineering and Technology International,Dec 6-8, 2013.

[7] J. Snchez and F. Aguayo, "Mobile messenger for the blind", in Proc. ECRIM '06, Springer, 2006, pp. 369385 .

[8] TuhinChakraborty and DebasisSamanta, "BlindGuide: An Audio Based Eyes-Free Caller Guide for People with Visual Impairment”, 27-29 Dec. 2012 IEEE Proceedings of 4th International Conference on Intelligent Human Computer Interaction, Kharagpur, India.
[9] PunyabrataGhatak, Mohan Singh, Chandan Kumar Ghoyal, SaurabhBanga, NeerajaAtri, "Swar-Suchak: Open source voice enabled information retrieval system", 3-5 June 2011 IEEE-International Conference on Recent Trends in Information Technology, ICRTIT.

[10] https://console.developers.Google.com/apis/api/maps_an droid_backend/overview?project=numeric-virtue-109512

[11] http://www.edumobile.org/android/androiddevelopment/get-location-from-gps/

[12] http://www.tutorialspoint.com/android/android_text_to_s peech.htm

[13] https://www.simplifiedcoding.net/android-sqlitedatabase-tutorial-insert-into-sqlite-db/ 